\title{
Single particle versus ensemble average: From power-law intermittency of a single quantum dot to quasistretched exponential fluorescence decay of an ensemble
}

\author{
Jau Tang ${ }^{\text {a) }}$ and R. A. Marcus ${ }^{\text {b) }}$ \\ Noyes Laboratory of Chemical Physics, California Institute of Technology, Pasadena, California 91125
}

(Received 20 June 2005; accepted 3 October 2005; published online 1 December 2005)

\begin{abstract}
Light-induced diffusion-controlled electron transfer is proposed as an underlying mechanism for the intermittency (power law and breakdown) of a single quantum dot and ensemble-averaged fluorescence decay. The intensity decay can be approximated to a stretched exponential expression. The physical links to the free energy gap, reorganization energy, electronic coupling, and diffusion correlation times are discussed. A procedure is described for extracting these molecular-based parameters from experiments and is demonstrated with examples using existing data.
\end{abstract}

(C) 2005 American Institute of Physics. [DOI: 10.1063/1.2128409]

\section{INTRODUCTION}

Quantum dots (QDs) as fluorescence tags and their potential applications in electronics and photonics have generated wide interest. ${ }^{1}$ Their fluorescence intermittency and decay have been investigated experimentally ${ }^{2-11}$ and theoretically. ${ }^{12-19}$ Blinking occurs when a QD, or a molecule in single-molecule spectroscopy, jumps stochastically between light and dark states. For ensemble-averaged fluorescence decay, there is no a priori knowledge of the history of an individual QD that undergoes numerous blinking cycles. Thus, to calculate fluorescence decay, which is equivalent to the sum of histograms from each QD in an ensemble, coupled equations with both forward and backward reactions are required.

Recently, we proposed a diffusion-controlled electron transfer (DCET) mechanism ${ }^{18}$ between a light state and a dark state for single QD dots. The diffusion is assumed to occur in energy space and represents energy fluctuation experienced by these two states. We considered a conduction band edge state in the band gap. The presence of a broad absorption spectrum for QDs indicates a quasicontinuum conduction band, but the presence of a very narrow emission spectrum indicates a very fast radiationless relaxation from the higher band states to the lowest exciton state that represents our photoexcited light state $\mid 1>$. In this model, the dark state is a charge-separated state with an electron trapped in a shallow surface state. There would be numerous surface states, delocalized over the surface, forming a thin band for dark QDs. In surface-enhanced emission on a rough gold surface, Shimizu et $a l^{7}$ observed binary hopping between two emission lines that are separated by about $2-25 \mathrm{meV}$. One of these two emission lines represents the ordinary emission line from the light state, and the other represents the surface-enhanced dark emission from the dark states. In addition, the dark emission is very narrow, indicating the

\footnotetext{
a)Electronic mail: jautang@caltech.edu

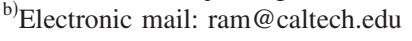

energy distribution for the dark surface states is not broad. Due to slow photoinduced structural changes, spectral diffusion is slow in QDs as observed by Empedocles et al. ${ }^{20} \mathrm{In}$ the present study we use this simplified two-state model with shallow energy traps $(<50 \mathrm{meV})$ which is distributed over the surface rather than being localized. We do not consider here deep trap states (several hundreds $\mathrm{meV}$ and $1 \mathrm{eV}$ ), which could cause intermittency via an Auger-assisted process. $^{21}$

In this paper, we investigate ensemble-averaged fluorescence decay, its distinction from the single particle behavior, and explain its relationship to single QD intermittency. We also show how molecular-based parameters can be extracted from the complementary experimental data, single QD intermittency data, and ensemble-averaged fluorescence decays.

\section{THE DIFFUSION-CONTROLLED ELECTRON TRANSFER MODEL}

The DCET mechanism is assumed to govern the charge transfer reactions between a light state $\mid 1>$ and a chargeseparated state $|2\rangle$ which appears dark due to a fast Auger relaxation process. Because $\mid 2>$ is a long-lived state, it is likely to be a charge-separated triplet state with a weak electronic coupling between the hole in the core of a QD and the surface-trapped electron. A triplet blinking mechanism has been invoked in an explanation of other single molecule fluorescence intermittency. ${ }^{22-24}$ Under continuous illumination, fast population recycling occurs between $|1\rangle$ due to a fluorescence decay rate $\gamma_{0}$ and population pumping of the ground state $\mid 0>$ at a rate $W$. Assuming nonadiabatic ET between $\mid 1>$ and $\mid 2>$, an initial Boltzmann population at the $\mid 0>$ prior to light illumination, the rate equation for the population $\rho_{k}(Q, t)$ for the $k$ th state $(k=0,1,2)$ at reaction coordinate $Q$ is given by ${ }^{19}$ 


$$
\begin{aligned}
\frac{\partial}{\partial t} \rho_{1}(Q, t)= & L_{1} \rho_{1}(Q, t)-\frac{2 \pi\left|V_{\mathrm{ex}}\right|^{2}}{\hbar} \delta\left[U_{12}(Q)\right]\left[\rho_{1}(Q, t)\right. \\
& \left.-\rho_{2}(Q, t)\right]-\gamma_{0} \rho_{1}(Q, t)+W \rho_{0}(Q, t), \\
\frac{\partial}{\partial t} \rho_{2}(Q, t)= & L_{2} \rho_{2}(Q, t)-\frac{2 \pi\left|V_{\mathrm{ex}}\right|^{2}}{\hbar} \delta\left[U_{12}(Q)\right] \\
& \times\left[\rho_{2}(Q, t)-\rho_{1}(Q, t)\right], \\
\frac{\partial}{\partial t} \rho_{0}(Q, t)= & \gamma_{0} \rho_{1}(Q, t)-W \rho_{0}(Q, t),
\end{aligned}
$$

where $L_{k}$ is the diffusion operator

$$
L_{k} \equiv \frac{\Delta_{k}^{2}}{\tau_{L, k}} \frac{\partial}{\partial Q}\left[\frac{\partial}{\partial Q}+\frac{1}{k_{B} T} \frac{\partial}{\partial Q} U_{k}(Q)\right] .
$$

With $W \ll \gamma_{0}$ and at time $t \gg 1 / W$, a quasiequilibrium is established between $\mid 0>$ and $\mid 1>$, Eq. (1a) can be approximated by

$$
\begin{aligned}
\frac{\partial}{\partial t} \rho_{2}(Q, t)= & L_{2, \mathrm{eff}} \rho_{2}(Q, t)-\frac{2 \pi\left|V_{k}\right|^{2}}{\hbar} \delta\left[U_{12}(Q)\right] \\
& \times\left[\rho_{2}(Q, t)-\rho_{1}(Q, t)\right]
\end{aligned}
$$

where $L_{k, \text { eff }} \equiv \zeta_{k} L_{k},\left|V_{k}\right|^{2} \equiv \zeta_{k}\left|V_{\text {ex }}\right|^{2}, \zeta_{1} \equiv W /\left(W+\gamma_{0}\right) \sim W / \gamma_{0}$, $\zeta_{2} \equiv 1, V_{\mathrm{ex}}$ the electronic coupling, $U_{12}(Q) \equiv U_{1}(Q)-U_{2}(Q)$, $U_{1}(Q)=\kappa\left(Q-Q_{0,1}\right)^{2} / 2$, and $U_{2}(Q)=\kappa\left(Q-Q_{0,2}\right)^{2} / 2+\Delta G^{0}$, $\kappa \Delta_{k}^{2}=k_{B} T$ at room temperature, $\lambda \equiv \kappa\left(Q_{0,1}-Q_{0,2}\right)^{2} / 2$ the reorganization energy, $\Delta G^{0}$ the free energy gap. Due to fast population recycling, the effective diffusion correlation time $1 / \tau_{1} \equiv W / \tau_{L, 1}\left(W+\gamma_{0}\right)$ depends on light intensity, the diffusion constant $\Delta_{1}^{2} / \tau_{1}$ of the light state increases with light intensity and is light driven. As suggested from the experiments of Empedocles et al., ${ }^{20}$ the spectral diffusion depends on light intensity and temperature, and their data were analyzed in our previous work. ${ }^{18}$ As pointed by Chung and Bawendi ${ }^{11}$ the diffusion for the dark state $\mid 2>$ can operate in darkness and may be enhanced with light illumination, and this diffusion and the light-driven diffusion in $\mid 1>$ lead to a steady state in ensemble measurements at very long time.

\section{A. Fluorescence intermittency of a single QD}

For a single QD, the lifetime probability distribution $P_{1}(t)\left[\right.$ or $\left.P_{2}(t)\right]$ for its fluorescence intermittency can be calculated from the decoupled forward (or reverse) rate equation. According to the previous paper, ${ }^{18}$ its Laplace transform is given by

$$
\bar{P}_{k}(s)=\frac{\bar{g}_{k}(s)}{1+\bar{g}_{k}(s)},
$$

where $\quad \bar{g}_{k}(s)=A_{k} \bar{G}_{k}\left(Q_{c}, Q_{c} ; s\right), \quad A_{k} \equiv(2 \pi / \hbar)\left|V_{k}\right|^{2} /$ $\left|\partial\left(U_{12}(Q)\right) / \partial Q\right|_{Q=Q_{c}}, Q_{c}$ is the energy level crossing point, i.e., $U_{12}\left(Q_{c}\right)=0$, and the Green function $\bar{G}_{k}\left(Q, Q^{\prime} ; s\right)$ satisfies $s \bar{G}_{k}\left(Q, Q^{\prime} ; s\right)-L_{k} \bar{G}_{k}\left(Q, Q^{\prime} ; s\right)=\delta\left(Q-Q^{\prime}\right)$. From Eq. (2a), one also has

$$
\bar{g}_{k}(s)=\frac{\bar{P}_{k}(s)}{1-\bar{P}_{k}(s)} .
$$

Defining $t_{c, k} \equiv 2 \lambda k_{B} T \hbar^{2} / \pi^{2} \tau_{k}\left|V_{k}\right|^{4}$ and $1 / \tau_{k} \equiv \zeta_{k} / \tau_{L, k}$ at very short time, when $t<t_{c, k}$ one obtains ${ }^{18,19}$

$$
P_{k}(t) \approx\left(1 / \sqrt{\pi t_{c, k}}\right) t^{-1 / 2},
$$

and the behavior later becomes

$$
P_{k}(t) \approx \sqrt{t_{c, k} / 4 \pi} t^{-3 / 2} \exp \left(-\Gamma_{k} t\right), \quad t_{c, k}<t<\tau_{k}, 1 / \Gamma_{k},
$$

where

$$
\begin{aligned}
& \Gamma_{k} \tau_{k} \equiv \frac{E_{A, k}}{2 k_{B} T}, \quad E_{A, 1} \equiv \frac{\left(\lambda+\Delta G^{0}\right)^{2}}{4 \lambda}, \\
& E_{A, 2} \equiv \frac{\left(\lambda-\Delta G^{0}\right)^{2}}{4 \lambda}, \\
& E_{A, 1}-E_{A, 2}=\Delta G^{0}, \quad 2 \Gamma_{1} \tau_{1}-2 \Gamma_{2} \tau_{2}=\frac{\Delta G^{0}}{k_{B} T} .
\end{aligned}
$$

The physical origin of the difference in the temporal behavior in Eqs. (3a) and (3b) was discussed previously. ${ }^{18}$ Equation (3b) shows that $P_{k}(t)$ follows a power law with an exponential tail. The bending factor $\Gamma_{1}$ (or $\Gamma_{2}$ ) derived earlier is related to the activation energy $E_{A, 1}$ for the forward (or $E_{A, 2}$ for reverse) electron transfer reaction as in Eq. (3c). Spectral diffusion of light QDs is light driven and temperature dependent. ${ }^{20}$ At higher light intensities or temperatures, or for QDs with a larger diameter (i.e., greater surface area for photoabsorption), the effective $1 / \tau_{1}$ (or $D_{k} / \Delta_{k}^{2}$ ) increases and so does $\Gamma_{1}$. Such dependence has been pointed out and extensively studied by Bawendi and coworkers. ${ }^{6,20}$

At a much longer time when $t \gg \tau_{k}$, one has $\bar{g}_{k}(s)$ $\approx \gamma_{k} / s+f_{k}$ at small $s$, and from Eq. (2a) one obtains

$$
P_{k}(t) \approx \gamma_{k, \mathrm{eff}} \exp \left(-\gamma_{k, \mathrm{eff}} t\right), \quad \gamma_{k, \mathrm{eff}}=\frac{\gamma_{k}}{1+f_{k}},
$$

where $\gamma_{1}\left(\right.$ or $\left.\gamma_{2}\right)$ is the conventional nonadiabatic forward (or reverse) electron transfer rate constant

$$
\gamma_{k}=\frac{2 \pi}{\hbar} \frac{\left|V_{k}\right|^{2}}{\sqrt{4 \pi \lambda k_{B} T}} \exp \left(-E_{A, k} / k_{B} T\right)
$$

and

$$
f_{k} \equiv \int_{0}^{\infty} d t \frac{A_{k} \exp \left(-2 \Gamma_{k} \tau_{L, k} \tanh \left(t / 2 \tau_{k}\right)\right)}{\sqrt{2 \pi \Delta_{k}^{2}\left(1-\exp \left(-2 t \tau_{k}\right)\right)}-\gamma_{k}} .
$$

Detailed evaluation of $f_{k}$ for various situations is given by Rips and Jortner. ${ }^{25}$

\section{B. Fluorescence decay of an ensemble of QDs}

In this study of ensemble-averaged fluorescence decay, Chung and Bawendi, ${ }^{11}$ measured the time evolution of fluorescence intensity with QDs initially in darkness prior to light illumination. The QDs are presumably in the ground state. If the Stokes shift between the ground state and the 
photoexcited light state $\mid 1>$ is small, as an approximation one can assume a Boltzmann distribution of configurations for $\mid 1>$. One obtains from Eq. (1c) by the Green function method the population $\left\langle\bar{\rho}_{1}(s)\right\rangle$, or the normalized fluorescence intensity $\bar{I}(s)$, assuming a quantum yield of 1 . In Laplace transform form it is

$$
\bar{I}(s) \equiv \int_{-\infty}^{\infty} d Q \bar{\rho}_{1}(Q, s)==\frac{\zeta_{1}}{s}\left\{1-\frac{\gamma_{1}}{s\left[1+\bar{g}_{1}(s)+\bar{g}_{2}(s)\right]}\right\},
$$

and at $t \gg \tau_{L, k}$ (very small $s$ ) Eq. (4a) yields

$$
\begin{aligned}
& I(t) / I(0) \sim I_{\mathrm{eq}}+\left(1-I_{\mathrm{eq}}\right) \exp \left(-t / T_{L}\right), \\
& 1 / T_{L} \equiv \frac{\gamma_{1}+\gamma_{2}}{1+f_{1}+f_{2}},
\end{aligned}
$$

where $I(0)=\zeta_{1}$ and

$$
I_{\text {eq }} \equiv 1 /\left[1+\zeta_{1} \exp \left(-\Delta G^{0} / k_{B} T\right)\right] \text {. }
$$

Using Eq. (2b), Eq. (4a) can also be expressed as

$$
\bar{I}(s)=\frac{\zeta_{1}}{s}\left\{1-\frac{\gamma_{1}\left[1-\bar{P}_{1}(s)\right]\left[1-\bar{P}_{2}(s)\right]}{s\left[1-\bar{P}_{1}(s) \bar{P}_{2}(s)\right]}\right\} .
$$

Margolin and Barkai ${ }^{17}$ introduced two-time correlation function $\left\langle I_{F}(t+\tau) I_{F}(t)\right\rangle /\left\langle I_{F}(t)\right\rangle\left\langle I_{F}(t+\tau)\right\rangle$, to analyze aging behavior of QDs, where $I_{F}(t)$ is unnormalized fluorescence intensity. It becomes $C_{F}(\tau) \equiv\left\langle I_{F}(t+\tau) I_{F}(t)\right\rangle /\left\langle I_{F}(t)\right\rangle^{2}$, if averaged over a sufficiently long period of time. $C_{F}(\tau)$ is often measured experimentally in study of QDs and other systems, ${ }^{9,13,17}$ and contains no $t$ depdendence and does not show aging of QDs. $\bar{I}(s)$ of Eq. (4d) is related to $\bar{C}_{F}(s)$ by

$$
\bar{I}(s)=\bar{C}_{F}(s) \zeta_{1} \gamma_{2} /\left(\gamma_{1}+\gamma_{2}\right)
$$

Equation (4d) expressed in terms of $\bar{P}_{k}(s)$ can be of practical use when one wants to calculate $\bar{I}(s)$ or $\bar{C}_{F}(s)$ from the phenomenological behavior of $\bar{P}_{k}(s)$ observed experimentally. The present work provides a physical link via Eqs. (2a) and (2b) with light-induced DCET as an underlying mechanism.

\section{ANALYSIS OF FLUORESCENCE DECAY DATA}

To analyze fluorescence data ${ }^{11}$ that usually extends from $1 \mathrm{~ms}$ to $10^{4} \mathrm{~s}$, one needs to calculate $I(t)$ from Eq. (4a) over the whole time span. The simple asymptotic approach often used for analyzing $P_{k}(t)$ is not sufficient for the entire time range. To cover a broad time range and a variety of experimental conditions as well as to develop approximate formulas, we evaluate Eq. (4a) numerically. $\bar{G}_{k}\left(Q_{c}, Q_{c} ; s\right)$ was evaluated for both small and large $s$ regimes using standard numerical inverse Laplace transform from IMSL. To obtain desired accuracy as many as 25 terms in Taylor's series expansion may be required if $\Gamma_{k} \tau_{k}>1$.

We have applied the results to the recent experimental data of Chung and Bawendi. ${ }^{11}$ In their measurements CdSe QDs of $25 \AA$ core radius with a ZnS shell were obtained at (a)

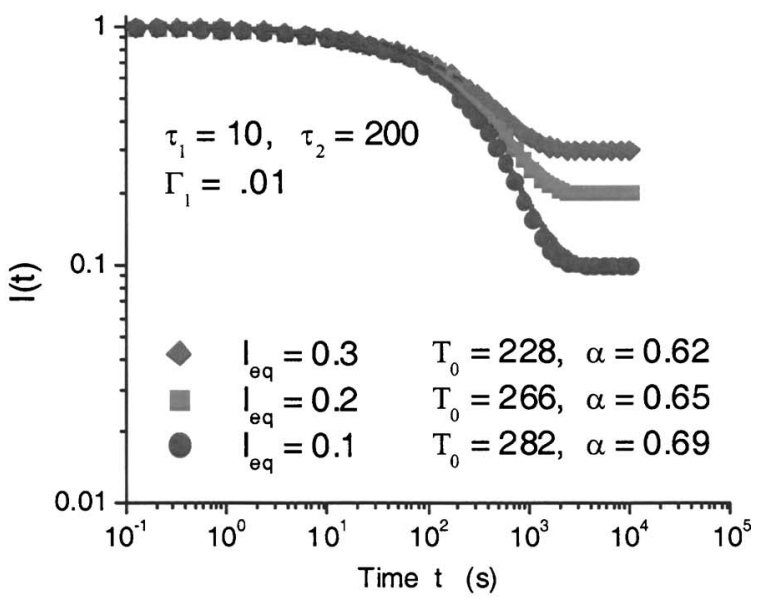

(b)

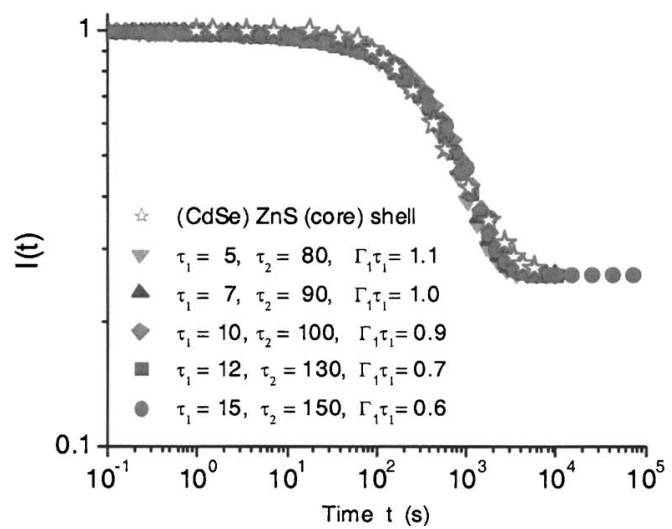

FIG. 1. (a) Log-log plot of the normalized $I(t)$ (dot curves) and the fitted (solid) curves using a stretched exponential $I_{\mathrm{eq}}+\left(1-I_{\mathrm{eq}}\right) \exp \left[-\left(t / T_{0}\right)^{\alpha}\right]$ with fitted values for $\alpha$ and $T_{0} . \tau_{1}=10 \mathrm{~s}, \tau_{2}=200 \mathrm{~s}$, and $t_{c, 1}=10^{-9} \mathrm{~s}$ were used. The value of $t_{c, 1}$ is presently unknown and could be much shorter but its choice is not critical. $I_{\mathrm{eq}}$ is varied with a fixed $\Gamma_{1}=0.01 \mathrm{~s}^{-1}$. (b) Experimental data of CdSe with a $\mathrm{ZnS}$ shell and fitted curves using Eq. (4a) with $I_{\text {eq }}$ $\sim 0.26$.

room temperature, with laser intensity about $2 \mathrm{~kW} / \mathrm{cm}^{2}$ with about 5000 QDs inside the focal areas for the ensemble averaging. With an absorption cross section ${ }^{26}$ of 4.6 $\times 10^{-16} \mathrm{~cm}^{2} \mathrm{eV}$ for $\mathrm{CdSe}(18 \AA)$ and a cubic dependence on the radius of a $\mathrm{QD},{ }^{27}$ we estimated the corresponding pumping rate $W \sim 1.5 \times 10^{7} \mathrm{~s}^{-1}$. With the fluorescence lifetime ${ }^{28}$ $1 / \gamma_{0} \sim 20 \mathrm{~ns}$, one has $\zeta_{1} \sim 0.75$. Although the diffusion of the light state depends on light intensity, the diffusion of the dark state can occur in dark, as pointed out by Chung and Bawendi. ${ }^{11}$ When the light is turned off, the remaining population in $\mid 2>$ can diffuse across the energy-level crossing and returns to $\mid 0>$ via $\mid 1>$ or via a slow radiationless transition to $\mid 0>$ which is neglected in this treatment.

As an illustration, the theoretical $I(t)$ from Eq. (4a) is shown in Fig. 1(a) with a fit using a stretched exponential. The experimental data ${ }^{11}$ are shown and fitted in Fig. 1(b) using theoretical curves from Eq. (4a). Another fit is shown in Fig. 2(a) using a stretched exponential $I_{\text {eq }}+\left(1-I_{\text {eq }}\right) \exp \left[-\left(t / T_{0}\right)^{\alpha}\right]$. The three-parameter stretched exponential fit $\left(T_{0}, \alpha\right.$, and $\left.I_{\mathrm{eq}}\right)$ is a convenient approximate representation for the overall decaying feature of $I(t)$. The 
(a)

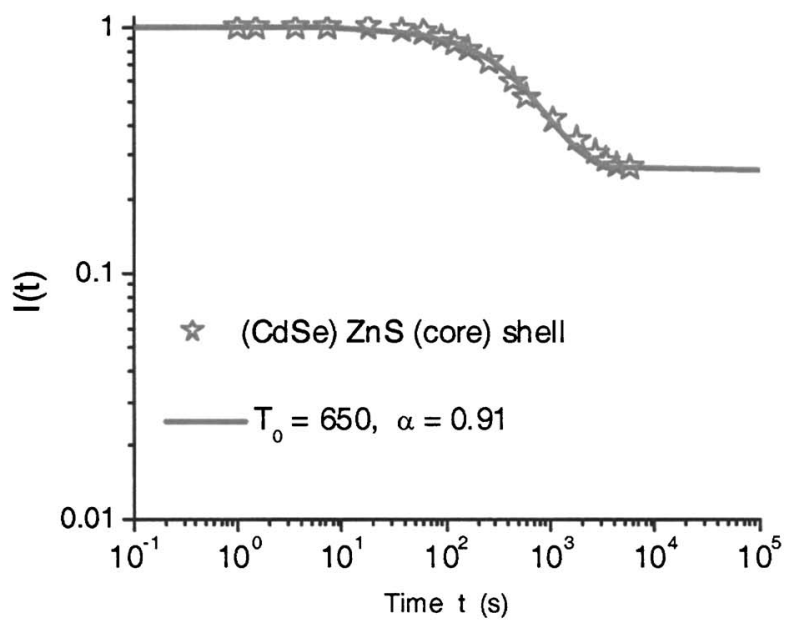

(b)

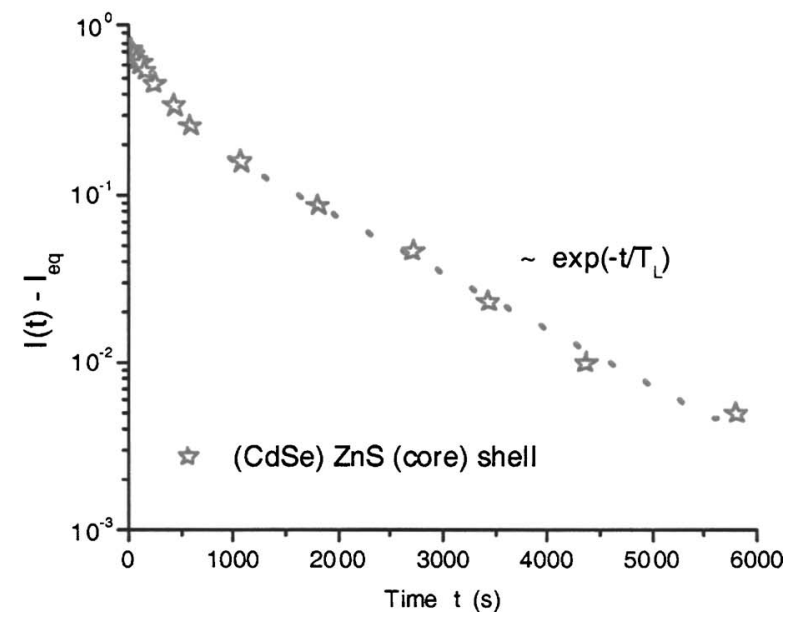

FIG. 2. (a) Experimental data approximated by a stretched exponential (solid line). (b) Long-time tail of $I(t)-I_{\mathrm{eq}}$ follows $\exp \left(-t / T_{L}\right)$ (dot line) where $T_{L} \sim 1300 \mathrm{~s}$

long-time tail of $I(t)-I_{\text {eq }}$ shown in Fig. 2(b) follows, instead, the theoretical single exponential decay $\exp \left(-t / T_{L}\right)$ of Eq. (4b), indicating the stretched exponential law is not exact but an approximation. Although the stretched exponential fit is less accurate in the tail section which is better fitted by a single exponential, it provides a quick estimate of relevant kinetic and energetic parameters. To be more accurate, one has to use the more elaborative Eq. (4a) that requires numerical inverse Laplace transform. As illustrated in Figs. 3(a) and 3(b) (showing all six curves merged for various parameter settings), we have examined the dependence of $T_{0}$ and obtained an empirical universal relation

$$
T_{0} \approx \frac{\left(\sqrt{\tau_{1}}+\sqrt{\tau_{2}}\right)^{2}}{\zeta_{1} \exp \left(-2 \Gamma_{1} \tau_{1}\right)+\zeta_{2} \exp \left(-2 \Gamma_{2} \tau_{2}\right)} .
$$

As illustrated in Fig. 4(a), the exponent $\alpha$ lies between 0.5 and 1. For $E_{A, k} / k_{B} T \gg 1, I(t)$ becomes a single exponential decay with $\alpha \sim 1$. Figure 4(b) shows all curves merged, and the dependence of $2 \alpha-1$ appears to follow an empirical universal relation: (a)

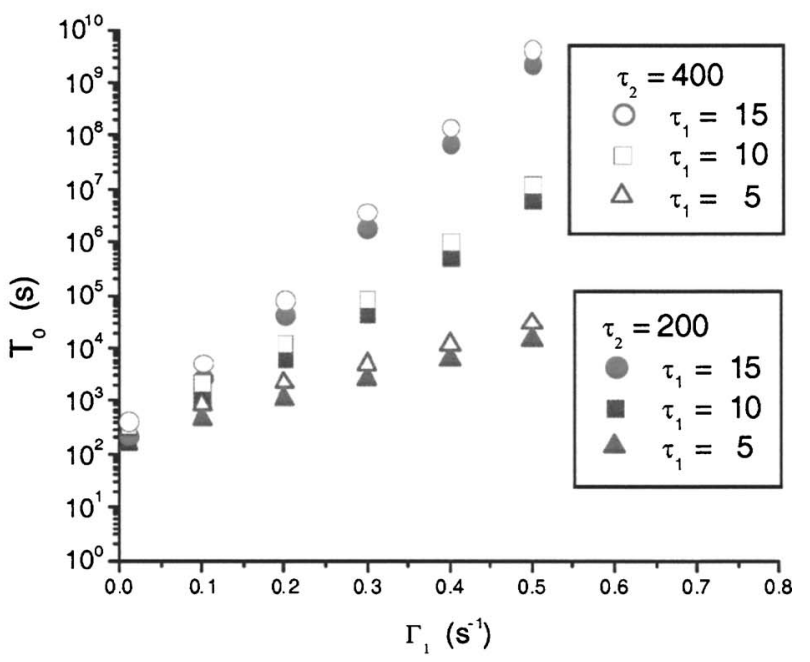

(b)

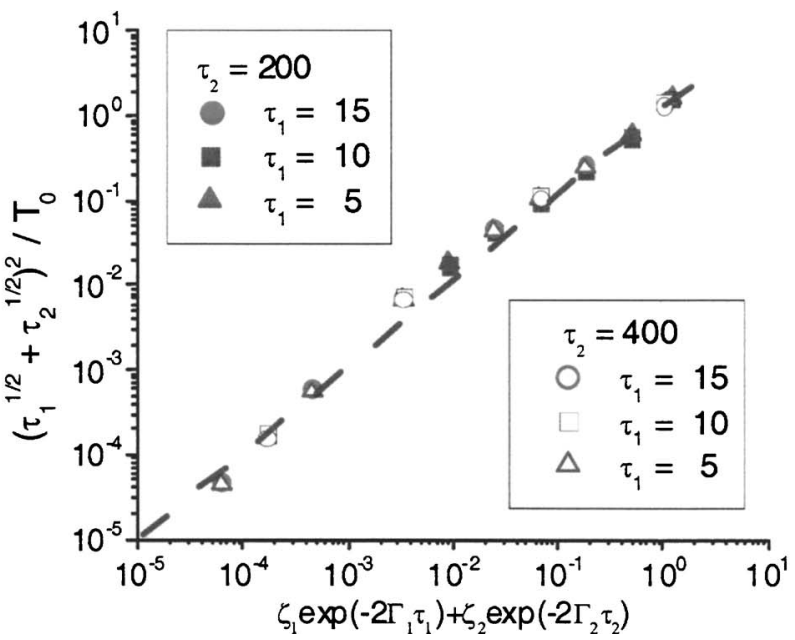

FIG. 3. (a) Semilog plot of $T_{0}$ vs $\Gamma_{1}$ for six parameter sets. (b) Universal dependence of $T_{0}$ according to Eq. (5) over 5 decades of changes. $2 \Gamma_{2} \tau_{2}=2 \Gamma_{1} \tau_{1}-\Delta G^{0} / k_{B} T$ was used in the equivalent expression.

$-\ln (2 \alpha-1) \approx \frac{2 \zeta_{1} \exp \left(-2 \Gamma_{1} \tau_{1}\right)+2 \zeta_{2} \exp \left(-2 \Gamma_{2} \tau_{2}\right)}{1+3 \tau_{1} / \tau_{2}}$.

Equations (5) and (6) link $T_{0}$ and $\alpha$ obtained from a stretched exponential fit to the data with kinetic parameters $\tau_{1}, \tau_{2}, \Gamma_{1}$, and $\Gamma_{2}$, with a constraint $\Gamma_{1} \tau_{1}-\Gamma_{2} \tau_{2}=\Delta G^{0} / 2 k_{B} T$. There are three parameters in a stretched exponential fit, $I_{\mathrm{eq}}$ (or $\Delta G^{0}$ ), $T_{0}$, and $\alpha$, can be determined from $I(t)$. Since there are four unknowns $\tau_{1}, \tau_{2}, \Gamma_{1}$, and $\Gamma_{2}$, other data are needed to uniquely determined them. A description for determining the molecular-based values and other related parameters is given in the following from systematic measurements of $I(t)$, $P_{1}(t), P_{2}(t)$ and spectral diffusion.

(0) Check if the phenomenology associated with the present DCET model is met. Is the exponent of the power law close to $-3 / 2$ ? Are $1 / \tau_{1}$ inferred from spectral diffusion and $\Gamma_{1}$ inferred from the bending tail proportional to light intensity? Is the steady-state $I_{\text {eq }}$ independent of light intensity? Only the first three of these conditions have been tested in experiments. 
(a)

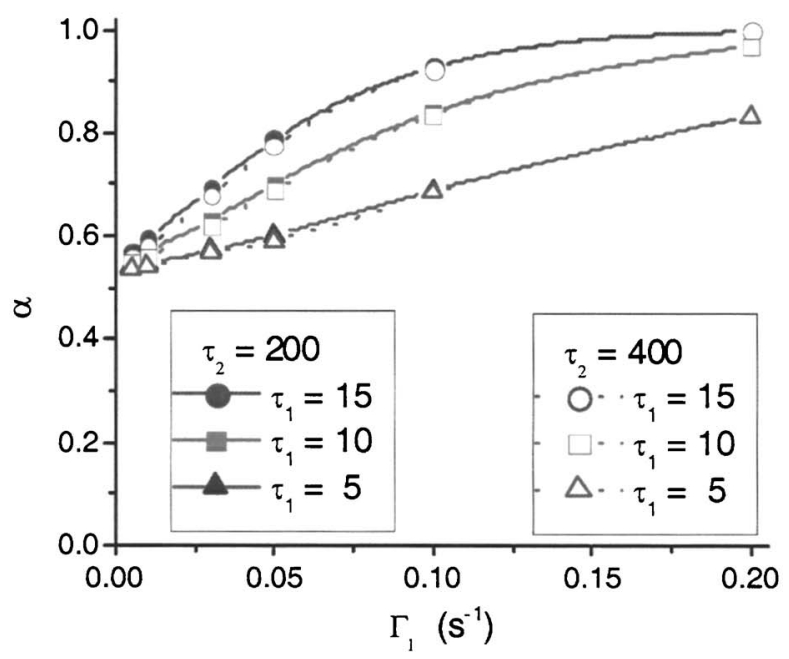

(b)

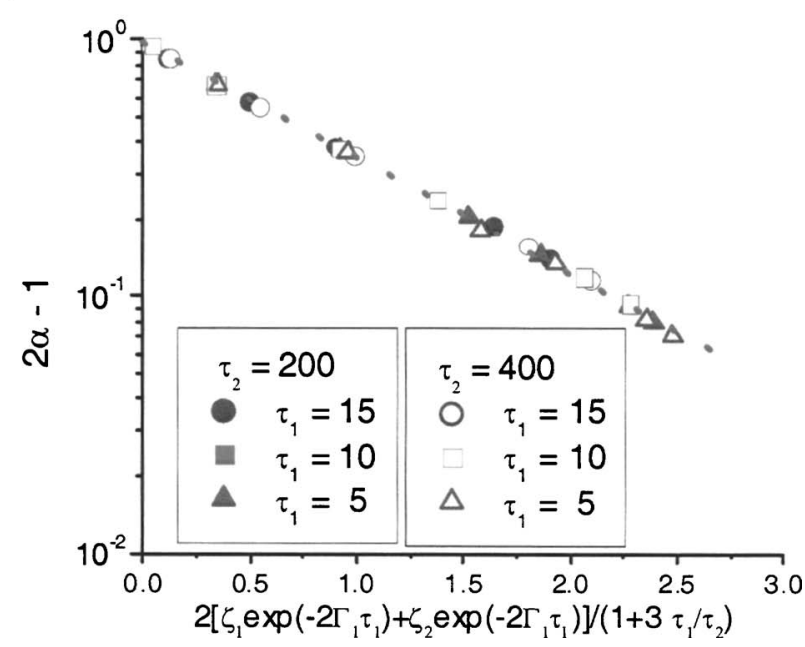

FIG. 4. (a) Plot of $\alpha$ vs $\Gamma_{1}$. (b) Semilog plot of $2 \alpha-1$ vs $2\left(\zeta_{1} \exp \right.$ $\left.\left(-2 \Gamma_{1} \tau_{1}\right)+\zeta_{2} \exp \left(-2 \Gamma_{2} \tau_{2}\right)\right) /\left(1+3 \tau_{1} / \tau_{2}\right)$, showing universal dependence over one decade. $I_{\text {eq }}=0.26$ was used in this example.

(1) Determine $\tau_{1}$ from the second moment ${ }^{18} \sigma^{2}(t)$ in spectral diffusion, ${ }^{20}$ using $\sigma^{2}(t) / \sigma^{2}(0)=1-\exp \left(-t / \tau_{1}\right)$.

(2) Determine $\Gamma_{1}$ from the exponential tail ${ }^{11}$ of the "light-period" intermittency $P_{1}(t)$ of a single QD. Because $\Gamma_{1}$ and $\tau_{1}$ are sensitive to light intensity, temperature and the size of QDs, the experimental conditions need to be specified.

(3) Determine $\Delta G^{0}$ from the steady-state value of the fluorescence decay $I(t)$ of a QD ensemble. ${ }^{11}$

(4a) Determine $T_{0}$ and $\alpha$ using a stretched exponential fit to $I(t) / I(0)$ with $I_{\text {eq }}+\left(1-I_{\text {eq }}\right) \exp \left[-\left(t / T_{0}\right)^{\alpha}\right]$ and then determine $\tau_{2}$ and $\Gamma_{2}$ using the empirical relations in Eqs. (5) and (6) with the values of $T_{0}$ and $\alpha$ extracted earlier.

(4b) The earlier step can be replaced by an alternative of using numerical inverse Laplace transform of Eq. (4a) to fit $I(t)$, or Eq. (4e) to fit the intensity autocorrelation function $C_{F}(t)$. From such a fit, one can determine $\tau_{2}$ and $\Gamma_{2}$. This alternative is more accurate than the simple fit with a stretched exponential but requires numerical solution of Eq. (4a).
TABLE I. Extraction of $\Delta G^{0}, \lambda, E_{A, 1}, E_{A, 2}, \tau_{1}, \tau_{2}, T_{L}, \Gamma_{1}$, and $\Gamma_{2}$.

\begin{tabular}{|c|c|c|}
\hline Parameters & Values & Experiments/equations \\
\hline$\Delta G^{0}$ & $-33 \mathrm{meV}$ & $I_{\mathrm{eq}}=1 /\left(1+\zeta_{1} \exp \left(-\Delta G^{0} / k_{B} T\right)\right)$ in Fig. 2(a) \\
\hline $1 / \Gamma_{1}$ & $11 \mathrm{~s}(\text { estimated })^{\mathrm{a}}$ & Intermittency $P_{k}(t) \sim t^{-3 / 2} \exp \left(-\Gamma_{k} t\right)$ \\
\hline$\Gamma_{1} \tau_{1}$ & 0.9 & Fitted $I(t)$ in Fig. 1(b) \\
\hline$\tau_{1}$ & $10 \mathrm{~s}(\text { estimated })^{\mathrm{a}}$ & From $\Gamma_{1} \tau_{1}$ and $\tau_{1}$ earlier \\
\hline$E_{A, 1}$ & $57 \mathrm{meV}$ & $E_{A, 1}=2 \Gamma_{1} \tau_{1} k_{B} T$ [Eq. (3c)] \\
\hline$E_{A, 2}$ & $52 \mathrm{meV}$ & $E_{A, 2}=E_{A, 1}-\Delta G^{0}[$ Eq. (3c) $]$ \\
\hline$\Gamma_{2} \tau_{2}$ & 1.42 & $2 \Gamma_{2} \tau_{2}=2 \Gamma_{1} \tau_{1}-\Delta G^{0} / k_{B} T$ [Eq. (3c)] \\
\hline$\tau_{2}$ & $100 \mathrm{~s}$ & Fitted $I(t)$ in Fig. 1(b) \\
\hline $1 / \Gamma_{2}$ & $70 \mathrm{~s}$ & From $\Gamma_{2} \tau_{2}$ and $\tau_{2}$ above \\
\hline$\lambda$ & 290 or $4 \mathrm{meV}$ & $\left(\lambda+\Delta G^{0}\right)^{2}-4 \lambda E_{A, 1}=0[$ Eq. $(3 \mathrm{c})]$ \\
\hline$T_{L}$ & $1300 \mathrm{~s}$ & Fitting $I(t)$ tail in Fig. 2(b) [Eq. (4b)] \\
\hline
\end{tabular}

${ }^{\mathrm{a}}$ To determine $\Gamma_{1}$ from bending tail and $\tau_{1}$ from spectral diffusion information about light intensity, temperature and the size of QDs are required. The earlier values for $\Gamma_{1}$ and $\tau_{1}$ are only estimates and need to be measured under the same experimental conditions as in ensemble fluorescence decay in order to accurately extract all other parameters.

(5) Determine $1 / T_{L}$, or $\left(\gamma_{1}+\gamma_{2}\right) /\left(1+f_{1}+f_{2}\right)$, from the long-time tail of $I(t)$ using Eq. (4b) for a consistency check of $\Delta G^{0}, \lambda, E_{A, 1}, E_{A, 2}$, etc.

(6) If one observes the early power law of Eq. (3a) at short times, determine the critical time constant $t_{c, k}$ that separates dual power law regimes. From it one can determine $V_{\mathrm{ex}}$. Observation of two-state intermittency at times shorter than the fluorescence lifetime may require ultrafast techniques such as upconversion.

Taking $1 / \Gamma_{1}=11 \mathrm{~s}$ as an example from the fit in Fig. 1(b), all relevant parameters are estimated and listed in Table I. Two possible solutions for $\lambda \sim 290$ and $4 \mathrm{meV}$ are obtained, corresponding to the normal and inverted regimes, respectively. Incidentally, the observed and theoretical Stokes shift between $\mid 0>$ and $\mid 1>$ is only a few $\mathrm{meV}^{29}$ and is consistent with our previous analysis ${ }^{18}$ using spectral diffusion data of Empedocles and Bawendi. ${ }^{20}$ As an approximation we neglected the Stokes shift and assumed the initial Boltzmann population distribution in the ground state in an ensemble prior to light illumination is preserved in the photoexcited state. Based on $\Delta G^{0} \sim-33 \mathrm{meV}$ as determined from $I_{\mathrm{eq}}$ in Fig. 1(b) and $\Gamma_{1} \tau_{1}=0.9$, the calculated $T_{0}$ $\sim 1030 \mathrm{~s}$ and $\alpha=0.85$ as compared to $650 \mathrm{~s}$ and 0.91 from a simple stretched exponential fit in Fig. 2(a).

\section{DISCUSSION AND CONCLUSIONS}

With DCET as an underlying mechanism, we have analyzed both single QD intermittency and ensemble fluorescence decay. This model leads to: (1) power law for intermittency of a single QD starts as $P_{k}(t) \sim t^{-1 / 2}$ at a much shorter time $\left(t<t_{c, k}\right)$; (2) at $t>t_{c, k}$ the power law becomes $P_{k}(t) \sim t^{-3 / 2}$; (3) at a later time it develops an exponential bending tail $t^{-3 / 2} \exp \left(-{ }_{k} t\right)$; (4) the latter finally turns into an exponential decay $\gamma_{k, \text { eff }} \exp \left(-\gamma_{k, \text { eff }} t\right)$ at still longer times; (5) the ensemble-averaged fluorescence intensity $I(t) / I(0)$ follows a nonexponential (approximated to stretched exponential) decay as $I_{\mathrm{eq}}+\left(1-I_{\mathrm{eq}}\right) \exp \left[-\left(t / T_{0}\right)^{\alpha}\right]$, except at very long times when it becomes a single exponential decay; (6) empirical universal laws for $T_{0}$ and $\alpha$ of stretched exponential 
decay; and (7) $I(t)$ becomes a single exponential decay at much longer time with $I(t) / I(0) \sim I_{\mathrm{eq}}+\left(1-I_{\mathrm{eq}}\right) \exp \left(-t / T_{L}\right)$. Links of the single particle and ensemble behavior to molecular-based parameters have been given earlier to $\Delta G^{0}$, $\lambda, E_{A, 1}, E_{A, 2}, \tau_{1}, \tau_{2}, \Gamma_{1}$, and $\Gamma_{2}$, and an estimate of these values has been obtained. The procedures of how these can be more systematically obtained from the data have been described.

In the present analysis, all rates involving the charge transfer are diffusion controlled for times longer than $t_{c, k}$. The existence of a potential well for the "light" state is supported, in the standard interpretation in the literature and in our interpretation, by the amplitude of spectral diffusion, an amplitude caused by fluctuations within a well. If a corresponding spectral diffusion is observed for the dark state, studied on a metal film which enhances the emission rate, then a well also exists for the dark state, and so the possibility of diffusion control also exists. Existing experimental results involve deep traps which are short lived. ${ }^{28}$ For internal consistency an explanation is needed as to why a deep trap state, is short-lived when a conduction band edge state is not. The deep trap state in CdSe is likely a hole in the surface Se dangling bond. ${ }^{30}$ Its short life suggests it is less stabilized by nuclear adjustments than is the conduction-edge band state.

In this study, the focus is on normal diffusion since Chung and Bawendi ${ }^{11}$ observed $t^{-1.5}$ power law in fluorescence intermittency of a single QD and the exponent of $-3 / 2$ is a hallmark of normal diffusion. ${ }^{18}$ Therefore, we also only consider normal diffusion in an ensemble QD system. In this DCET model, for simplicity $|2\rangle$ represents a chargeseparated state with an electron in a shallow delocalized surface state. The energy fluctuation at the surface state can be caused by stochastic environmental changes or migration of the trap state to others at a slightly different energy, similar to a band structure for the surface states. If so, one can average Eq. (4a) over a distribution of free energy $\Delta G^{0}$, as is done in a study of electron transfer to a continuum of levels in metal or semiconductor surfaces, ${ }^{31}$ and so obtain an effective two-state scheme.

A recent study by Issac et $a l^{32}$ indicates the exponent for the power law may be correlated with dielectric properties. This DCET model with a non-Debye dielectric medium with non-Markovian diffusion was discussed in our previous work, ${ }^{19}$ showing the exponent of the power law is no longer $-3 / 2$ but could be between -1 and -2 . For the ensemble system, the factor $\alpha$ of the stretched exponential is expected to be different from the ideal Markovian case. These modifications could also affect the empirical universal laws given in Eqs. (5) and (6). Because anomalous diffusion is much more complex and would require lengthy description. In the present study we have also made an approximation in assuming an initial Boltzmann distribution in $|1\rangle$ soon after the light illumination is turned on. If it were non-Boltzmann, Eqs. (4a) or (4d) would need to be modified. More details will be presented elsewhere.

\section{ACKNOWLEDGMENTS}

The authors acknowledge the support of the National Science Foundation and the Office of Naval Research. J.T. also thanks the support by the James W. Glanville Foundation at the California Institute of Technology. J.T. benefited from a discussion with Dr. Chung and Professor Bawendi about their experiments that are central to this study.

${ }^{1}$ W. J. Parak, T. Pellegrino, and C. Plank, Nanotechnology 16, R9 (2005).

${ }^{2}$ M. Nirmal, B. O. Dabbousi, M. G. Bawendi, J. J. Macklin, J. K. Trautman, T. D. Harris, and L. E. Brus, Nature (London) 383, 802 (1996).

${ }^{3}$ M. Kuno, D. P. Fromm, H. F. Hamann, A. Gallagher, and D. J. Nesbitt, J. Chem. Phys. 112, 3117 (2000).

${ }^{4}$ M. Kuno, D. P. Fromm, A. Gallagher, D. J. Nesbitt, O. I. Micic, and J. J. Nozik, Nano Lett. 1, 557 (2001).

${ }^{5}$ M. Kuno, D. P. Fromm, H. F. Hamann, A. Gallagher, and D. J. Nesbitt, J. Chem. Phys. 115, 1028 (2001).

${ }^{6}$ K. T. Shimizu, R. G. Neuhauser, C. A. Leatherdale, S. A. Empedocles, W. K. Woo, and M. G. Bawendi, Phys. Rev. B 63, 205316 (2001).

${ }^{7}$ K. T. Shimizu, W. K. Woo, B. R. Fisher, H. J. Eisler, and M. G. Bawendi, Phys. Rev. Lett. 89, 117401 (2002).

${ }^{8}$ X. Brokmann, J. P. Hermier, G. Messin, P. Desbiolles, J. P. Bouchaud, and M. Dahan, Phys. Rev. Lett. 90, 120601 (2003).

${ }^{9}$ R. Verberk, A. M. van Oijen, and M. Orrit, Phys. Rev. B 66, 233202 (2002).

${ }^{10}$ M. Kuno, D. P. Fromm, S. T. Johnson, A. Gallagher, and D. J. Nesbitt, Phys. Rev. B 67, 125304 (2003)

${ }^{11}$ I. Chung and M. G. Bawendi, Phys. Rev. B 70, 165304 (2004).

${ }^{12}$ J. Wang and P. Wolynes, J. Chem. Phys. 110, 4812 (1999).

${ }^{13}$ M. Boguna, A. M. Berezhkovskii, and G. H. Weiss, Physica A 282, 475 (2000).

${ }^{14}$ Y. Jung, E. Barkai, and R. J. Silbey, Chem. Phys. 284, 181 (2002).

${ }^{15}$ R. Verberk and M. Orrit, J. Chem. Phys. 119, 2214 (2003).

${ }^{16}$ E. Barkai, Y. Jung, and R. Silbey, Annu. Rev. Phys. Chem. 55, 457 (2004).

${ }^{17}$ G. Margolin and E. Barkai, J. Chem. Phys. 121, 1566 (2004).

${ }^{18}$ J. Tang and R. A. Marcus, J. Chem. Phys. 123, 054704 (2005).

${ }^{19}$ J. Tang and R. A. Marcus, Phys. Rev. Lett. 95, 107401 (2005).

${ }^{20}$ S. A. Empedocles and M. G. Bawendi, J. Phys. Chem. B 103, 1826 (1999).

${ }^{21}$ P. A. Frantsuzov and R. A. Marcus, Phys. Rev. B 82, 155321 (2005).

${ }^{22}$ M. Hasse, C. G. Hübner, E. Eeuther, A. Herrmann, K. Müllen, and T. Basche, J. Phys. Chem. B 108, 10445 (2004).

${ }^{23}$ R. Zondervan, F. Kulzer, S. B. Orlinskii, and M. Orrit, J. Phys. Chem. A 107, 6770 (2003)

${ }^{24}$ J. Schuster, F. Cichos, and C. von Borczyskowski, Appl. Phys. Lett. 87, 051915 (2005).

${ }^{25}$ I. Rips and J. Jortner, J. Chem. Phys. 87, 6513 (1987).

${ }^{26}$ L. Lounis, H. A. Bechtel, D. Gerion, P. Alivisatos, and W. E. Moerner, Chem. Phys. Lett. 329, 399 (2000).

${ }^{27}$ C. A. Leatherdale, W. K. Woo, F. V. Mikulec, and M. G. Bawendi, J. Phys. Chem. B 106, 7619 (2002).

${ }^{28}$ G. Schlegel, J. Bohnenberger, I. Potapova, and A. Mews, Phys. Rev. Lett. 88, 137401 (2002)

${ }^{29}$ D. F. Underwood, T. Kippeny, and S. Rosenthal, J. Phys. Chem. B 105, 346 (2001).

${ }^{30}$ J. J. Shiang, A. V. Kadavanich, R. K. Grubbs, and A. P. Alivisatos, J. Phys. Chem. 99, 17417 (1995).

${ }^{31}$ S. Gosavi, Y. Q. Gao, and R. A. Marcus, J. Electroanal. Chem. 500, 7177 (2001); S. Gosavi and R. A. Marcus, J. Phys. Chem. B 104, 2067 (2000).

${ }^{32}$ A. Issac, C. von Borczyskowski, and F. Cichos, Phys. Rev. B 71, 161302(R) (2005) 